\title{
Matter, Dark Matter and Quartic Potential Generated by Unstable Confinement of Quarks
}

\author{
Leif Matsson \\ Department of Physics, University of Gothenburg, Gothenburg, Sweden \\ Email: leif.matsson@telia.com
}

How to cite this paper: Matsson, L. (2021) Matter, Dark Matter and Quartic Potential Generated by Unstable Confinement of Quarks. Journal of Modern Physics, 12, 19221929.

https://doi.org/10.4236/jmp.2021.1213109

Received: October 21, 2021

Accepted: November 27, 2021

Published: November 30, 2021

Copyright (อ 2021 by author(s) and Scientific Research Publishing Inc. This work is licensed under the Creative Commons Attribution International License (CC BY 4.0).

http://creativecommons.org/licenses/by/4.0/

\begin{abstract}
The Standard Model for particle physics is here extended by making a nonequilibrium filling of the empty vacuum after the start of Big Bang. The process is described as an unstable binding of massless quarks to massless antiquarks. When the filled part of vacuum condenses, the system becomes stabilized, quarks acquire mass and become confined and a quartic potential is induced, which hence need not be introduced ad hoc. The coupling and scale parameters in this potential have become asymmetric microscopic functions of the quark and antiquark densities. The so obtained dynamics can explain how the matter-antimatter asymmetry in the Universe and dark matter emerged. Quantum corrections are included and the model then gives ordinary matter, dark matter and dark energy contents at correct orders of magnitude.
\end{abstract}

\section{Keywords}

Dark Matter, Dark Energy, Cosmology, Quartic Potential

\section{Introduction}

It has been thought that Electroweak (EW) baryogenesis and QCD confinement could help to explain how the Standard Model (SM) emerged [1]-[7]. However, many questions have remained open and many new problems have emerged. For instance, the lambda cold dark matter (LCDM) model postulates that the expansion of the Universe is driven by dark energy (DE) and dark matter (DM), and that the galaxy structures we see today are due to density variations in the very early Universe. Such variations are expected to produce gravitational waves and thus a signal in the cosmic microwave background. However, such a signal has not been observed [8]. This is not a problem in the model suggested here, because it starts from a completely empty vacuum. 
The generation of matter and dark matter is in this model induced by a non-equilibrium filling of vacuum which is here assumed to have been totally empty at the Big Bang. Big Bang is assumed to have started at infinite energy conditions from a completely empty vacuum and that the Universe then contained equal and isotropic densities of free massless quarks and antiquarks, leptons and antileptons. At lower energies, the QCD model appears to remain intact with a stable vacuum even if a finite number of quark-antiquark pairs are generated. However, above a finite critical energy (temperature) $E_{\odot}$ surplus quarks (and surplus leptons) became generated, implying that vacuum could then not be expected to have been stable enough for quantum field theories to have been valid. The non-equilibrium filling of vacuum has another advantage. It generates the quartic (so called Ginsburg Landau or Higgs) potential, which thus need not be assumed in an ad hoc manner as in the SM.

The Dirac equation [9] and QED did not have any vacuum problems, because these models were not used at very high energies. As demonstrated before [10], surplus quarks were generated by a non-equilibrium filling of the empty QCD vacuum, a process which had to be described by classical fields. The non-equilibrium filling of vacuum, followed by condensation (assumed to have occurred when the energy decreased after Big Bang), generated a quartic potential, in which the coupling $\lambda=g^{2}$ and the scale $a$ became asymmetric microscopic functions of the quark and antiquark densities.

The density of surplus quarks in the numerator of the coupling $g$ in this model is proportional to the density of nucleons and hence approximately to the density of ordinary mass in the Universe. Except for this density of surplus quarks, the denominator of $g$ also contains the two equal densities of non-surplus quarks and antiquarks, which are here interpreted as building blocks of dark matter (DM). It is assumed here that top quarks dominated at higher energies already from the start of Big Bang, but all types of quarks should give the same result. It is not known how fast the maximal density of surplus quarks, corresponding to the actual density of protons in the Universe, is attained. However, the nonquantal vacuum filling process should be normalized such that $\lambda=g^{2}=0.11$ at $E_{c}=10^{5}$ $\mathrm{GeV}$ (Figure 1), where vacuum is sufficiently filled for the standard model to start working. At energies lower than $10^{5} \mathrm{GeV}$, the matter-DM ratio then approaches the value observed.

Leptons are assumed to contribute similarly, but much less, and are therefore neglected here. Under assumption that confinement started as a non-equilibrium binding of massless quarks, color by color, the suggested model yields observable matter, DM and DE contents at correct orders of magnitude. The results obtained show that the critical energy level $E_{c}$ is much below $10^{10} \mathrm{GeV}$ at which $\lambda_{\text {quartic }}$ in the SM becomes negative [11].

\section{Unstable Confinement after Big Bang}

After the start of Big Bang, an infinite number of the empty negative energy va- 
cuum states below the critical vacuum level $-E_{c}$ must have been filled before quantum effects could be expected to have had any impact. The non-equilibrium filling of this part of the QCD vacuum, which started at infinite energy conditions, could be viewed as a strong but unstable binding of massless quarks to antiquarks

$$
\frac{\mathrm{d} \psi}{\mathrm{d} t}=k \cdot \rho(x, t) \cdot \rho_{\alpha}(x, t)-k^{\prime} \psi(x, t),
$$

where $\rho, \rho_{\alpha}$ and $\psi$ are classical fields (densities) of quarks, antiquarks and quarkantiquark pairs, and $k$ and $k^{\prime}$ are the association and dissociation constants. This form of confinement process is here assumed to have been equal for all flavours and colours. The filling of the remaining finite number of vacuum states above $-E_{c}$ (energies below $E_{c}$ ), is sufficiently stable to be described as usual in the SM.

By insertion of the initial constraints, $\rho(x, t)=\rho_{0}-\psi(x, t)$ and $\rho_{a}(\boldsymbol{x}, t)=\rho_{a 0}-\psi(\boldsymbol{x}, t)$, where $\rho_{0}$ and $\rho_{a 0}$ are the two equal initial densities of quarks and antiquarks, here assumed to be equal, Equation (1) becomes

$$
\frac{\mathrm{d} \psi}{\mathrm{d} t}=k \cdot\left((a-\psi(\boldsymbol{x}, t))^{2}-\left(a^{2}-b^{2}\right)\right),
$$

in which $a=\left(\rho_{0}+\rho_{a 0}+K\right) / 2, b^{2}=\rho_{0} \rho_{a 0}$, and $K=k^{\prime} / k$. Integration of Equation (2) then yields

$$
\ln \left(\frac{a+\sqrt{a^{2}-b^{2}}-\psi}{a-\sqrt{a^{2}-b^{2}}-\psi} \frac{a(1-g)}{a(1+g)}\right)=\ln \left(\frac{\rho_{K}(x, t)}{\rho_{\alpha K}(x, t)} \frac{\rho_{\alpha K}}{\rho_{K}}\right)=2 \mathrm{kag} \cdot\left(t-t_{0}\right)
$$

where $\rho_{K}(x, t)=\rho_{K}-\psi(x, t)$ and $\rho_{a K}(x, t)=\rho_{a K}-\psi(x, t)$ are the time dependent quark and antiquark densities. With $\rho_{K}+\rho_{a K}=2 a$ and $g=\sqrt{\left(a^{2}-b^{2}\right) / a^{2}}=\left(\rho_{K}-\rho_{a K}\right) /\left(\rho_{K}+\rho_{a K}\right)>0$ the corresponding after start 'new initial' densities $\rho_{K}$ and $\rho_{\alpha K}$ can be written as $\rho_{K}=a(1+g) \geq \rho_{0}$ and $\rho_{a K}=a(1-g) \leq \rho_{a 0}$. For $k^{\prime}>0$ the coupling $g$ thus becomes an asymmetric microscopic function of the quark and antiquark densities, which shows that the suggested model works like a seesaw.

At the start of Big Bang, the Universe contained equal densities $\rho_{0}$ and $\rho_{a 0}$ of free massless quarks and antiquarks, which might then have condensed pairwise, $\left(\rho \rho_{a}\right)\left(\rho \rho_{a}\right)\left(\rho \rho_{a}\right) \ldots$, without generating any surplus quarks. But as described in Equation (3), the quarks confined asymmetrically, without leaving any antiquarks behind, into a system, which apart from pairs of non-surplus quarks $\rho_{N S}$ and antiquarks $\rho_{a K}$, contained surplus quarks $\rho_{s}, \rho_{s}\left(\rho_{a K} \rho_{N s}\right)\left(\rho_{a K} \rho_{N s}\right)\left(\rho_{a K} \rho_{N s}\right) \ldots$ This still implies that the non-surplus quarks were equally many as the antiquarks, which they were also before the start of Big Bang. However, it should be observed that at infinite energy (temperature), before the confinement had started, all pairs $\left(\rho \rho_{a}\right)$ were free and there was no difference between the two systems.

The numerator of the coupling $g$ equals the density of surplus quarks which can also be separated out in the denominator of $g$. Apart from that the denominator of $g=\rho_{s} /\left(\rho_{s}+\rho_{N s}+\rho_{a K}\right)$ also contains the two equal densities of non-surplus quarks $\rho_{N s}$ and antiquarks $\rho_{\alpha K}$, which are here interpreted as building 
blocks of DM and black holes. When the Universe cooled down to the critical energy level $E_{\diamond}$ the strong binding between non-surplus quarks and antiquarks started to become stable. The coupling $g$ then increased above $1 /(1+1+1) \approx 0.33$. Surplus leptons are expected to have emerged similarly.

\section{A Microscopic Form of Potential}

After binding into quark-antiquark pairs, this system condensed. This process can be described by a change of variable

$$
\varphi(x, t)=\frac{\sqrt{a^{2}-b^{2}}}{1-\psi(x, t) / a}
$$

which can also be viewed as a partition function where $\psi /$ a plays a role as non-equilibrium "fugacity" driven by Equation (2).

The derivative of Equation (4) combined with Equation (2) gives

$$
\frac{\mathrm{d} \varphi}{k \mathrm{~d} t}=\frac{\mathrm{d} \varphi}{\mathrm{d} x}=g\left(a^{2}-\varphi^{2}\right),
$$

which describes the condensation of the strongly bound $\rho \rho_{a}$ - pairs as a travelling wave $\varphi(x)=a \tanh (\operatorname{ag} x)$ that propagates at a velocity $k=x / t$.

The square of Equation (5) yields a microscopic form of the quartic potential $V(\varphi)$

$$
\frac{1}{2}\left(\frac{\mathrm{d} \varphi}{\mathrm{d} x}\right)^{2}=\frac{g^{2}}{2}\left(a^{2}-\varphi^{2}\right)^{2} \equiv V(\varphi),
$$

which hence need not be inserted ad hoc like in electroweak theory, and in which both the scale $a$ and the coupling $g$ have become microscopic functions of the quark and antiquark densities. As will be shown, after inclusion of quantum corrections below $E_{\varsigma}$, the microscopic potential $V(\varphi)$ approaches the quartic potential in the EW theory when the energy decreases further.

By breakdown of symmetry, $\varphi \rightarrow-a+\varphi$, Equation (6) then gives the equation of motion

$$
\frac{1}{2}\left(\frac{\mathrm{d} \varphi}{\mathrm{d} t}\right)^{2}-\frac{1}{2}\left(\frac{\mathrm{d} \varphi}{\mathrm{d} x}\right)^{2}+(2 g a)^{2} \varphi=6 \lambda \varphi^{2}(a-\varphi / 3)
$$

where $2 g a=m_{B}$ is the mass of a boson, a precursor to the Higgs boson, and $\lambda$ $=g^{2}$. It is not known exactly when the condensation started and at what rate, i.e. if the symmetry breakdown occurred exactly at $10^{5} \mathrm{GeV}$.

\section{Quantum Corrections}

In the previous "classical" approach [10], 2ga was identified as the mass of the Higgs boson $m_{H} \approx 125 \mathrm{GeV}$. With $a=\rho_{0} \approx 174 \mathrm{GeV}$ and $g \approx m_{H} / 348=0.36$, the coupling $\lambda=g^{2}=0.13$ was then interpreted as the matter to all matter ratio (except dark energy), as if quantum effects were already included. However, in this work $2 g a=m_{B}$ is identified as the mass of the becoming Higgs particle, a precursor boson that gets the mass, $m_{H}=125 \mathrm{GeV}$, at a certain critical energy 
below $E_{c}$ at which quantum effects started to contribute. However, before the energy has decreased to $E_{\circ}$, the density of surplus quarks must have increased from zero to the value at which quantum effects start to contribute.

The problem is then to combine the result of the nonequilibrium filling of vacuum, which had to be described in the terms of classical fields, with the finite energy result of QCD quantum field theory [11], and to determine the critical energy level $E_{c}$ at which this happens. But in order to simplify this task, the scale $a$ is first modified from $174 \mathrm{GeV}$ to the level of the top quark mass $m_{\text {top }}=173 \mathrm{GeV}$.

The mass $m_{H}$ in the RG improved $m_{H} / m_{\text {top }}$ - ratio at different energies in Fig. 2 in ref. [11] is here interpreted as the mass of the precursor boson $m_{B}$ in Equation (7). This mass changes with energy such that the $m_{H} / m_{\text {top }}$ - ratio attains $125 / 173$ $\approx 0.72$, and the numerator thus attains the mass of the Higgs boson, at about (or somewhat below) $10^{3} \mathrm{GeV}$ in Fig 2 in ref. [11]. By inclusion of quantum effects below $10^{5} \mathrm{GeV}$ in the model suggested here the $m_{B} / m_{\text {top }}$ - ratio thus first increases from 0 to 0.66 , and according to Fig. 2 in ref. [11], it then increases to $0.68,0.72$, 0.77 when the energy decreases further to $10^{4}, 10^{3}, 10^{2} \mathrm{GeV}$. Given these numbers of the $m_{B} / m_{\text {top }}$ - ratio and $m_{\text {top }}=173 \mathrm{GeV}$, the boson mass $m_{B}$ then correspondingly becomes $114.18,117.64,124.56,133.21 \mathrm{GeV}$ at the actual energy levels.

The coupling constant $g=m_{B} / 2 a$ thus first increases from 0 to $0.33=$ 114.18/346 and hence $\lambda=g^{2}=0.11$ at $10^{5} \mathrm{GeV}$ in Figure 1 is thus reached from below when the energy decreases from higher energies.

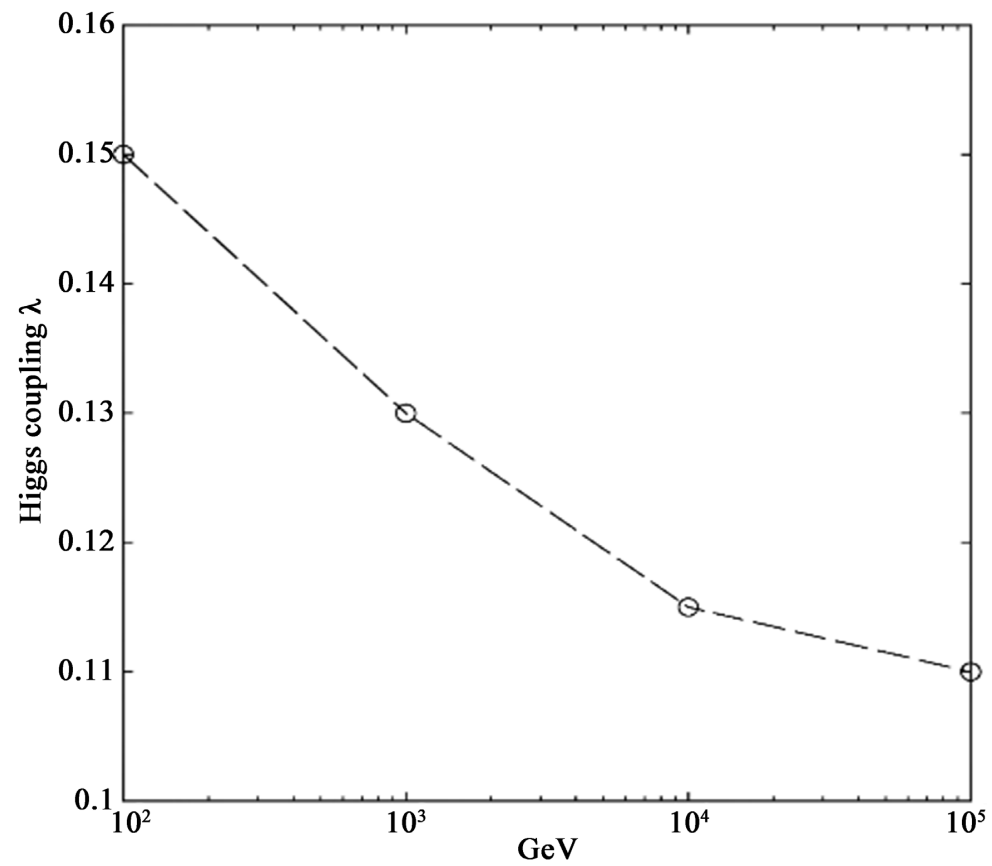

Figure 1 . The coupling $\lambda=g^{2}$ as a function of energy.

The coupling $g$ then increases from 0.330 to $0.340,0.360,0.385$, and $\lambda=g^{2}$ correspondingly becomes $0.109,0.116,0.130,0.148$ (see Figure 1 here) at the 
actual energy levels. Accordingly, $\lambda=(125 / 346)^{2}=m_{H} / 2 a=0.13$ at $10^{3} \mathrm{GeV}$ and $\lambda=0.148 \approx 0.15$ at $10^{2} \mathrm{GeV}$. However, the $m_{H} / m_{W}$ - ratio in Fig 2 in ref. [11] shows that $m_{H} / m_{W}=1.55 \approx 124.56 / 80.40$, hence, $m_{H} \approx 125 \mathrm{GeV}$ is reached already at $3.46 \times 10^{2} \mathrm{GeV}$. The start of impact of quantum corrections should thus be correspondingly delayed from $10^{5} \mathrm{GeV}$ to $3.46 \cdot 10^{4} \mathrm{GeV}$.

It seems that the Universe has always remembered its quantum origin [12 14], from the start of Big Bang at infinite energy when vacuum was totally empty, until it became sufficiently filled for quantum field theories (QFTs) in the SM to start working. But as described here, for the density of quarks $\rho_{s}=\left(\rho_{K}-\rho_{\alpha K}\right)$ (and correspondingly for leptons) to increase from zero to a critical level corresponding to the amount of observable matter in the Universe, the filling of vacuum must have been a non-equilibrium process. This cannot be explained by the SM, which only allows the creation or annihilation of a finite number of particle-antiparticle pairs.

On the contrary, in the model suggested here the numerator of the coupling $g$ describes the gap between the densities of quarks $\rho_{s}$ and antiquarks $\rho_{a k}$. After separation of the density of quarks $\rho_{K}$ in the denominator of $g$ into surplus quarks $\rho_{s}$ and non-surplus quarks $\rho_{N s}$ the density of non-surplus quarks $\rho_{N s}$ becomes equal to the density of antiquarks $\rho_{\alpha K}$

$$
g=\left(\rho_{K}-\rho_{a K}\right) /\left(\rho_{K}+\rho_{a K}\right)=\rho_{s} /\left(\rho_{s}+\rho_{N s}+\rho_{a K}\right)>0 .
$$

Quantum effects are here assumed to have started to contribute at $10^{5} \mathrm{GeV}$ where $g$ was first equal to $1 /(1+1+1)=1 /(1+2)=1 / 3$ and $\lambda=g^{2} \approx 0.11$ (Figure 1). The massive non-surplus quarks $\left(\rho_{N s} \approx 1\right)$ and antiquarks $\left(\rho_{a K} \approx 1\right)$ then formed more strongly bound states of dark matter, and the denominator of $g$ then decreased below 3 . As already explained, the coupling $\lambda$ then increased successively from $0.109 \approx 0.11$ to $0.148 \approx 0.15$, see Figure 1 .

As is clear from eq. (8), the coupling $g$ and hence also $\lambda=g^{2}$ define the relationship between matter and DM. Accordingly, $0.148=0.049 / 0.33$ corresponds to the ratio between matter and the sum of matter and DM. The corresponding relative content of DM in the Universe thus becomes $0.33-0.049=0.28$, and the remaining $67 \%$ corresponds to the density of dark energy, which also corresponds to the value of the cosmological constant. The cosmological constant has thus decreased from 1 to 0.67 during the generation of $28 \% \mathrm{DM}$.

\section{Summary}

Infrared divergence is but one reason why quark confinement cannot be described perturbatively in QCD [15]. However, as demonstrated here, in Equation (1), the confinement can be described non-perturbatively, and since the density of quarks must increase over that of antiquarks, this vacuum filling must be a non-equilibrium process. A second reason is that quantum fields lose their definition due to vacuum instabilities above the critical energy $E_{\odot}$ after emptying of a finite number of vacuum states (creation of a finite number of quark-antiquark pairs). The suggested model thus described the non-equilibrium dynamics above 
$E_{c}$ that generated surplus quarks, how this is connected with the QCD part of the $\mathrm{SM}$, and it also explained the generation of the quartic potential, the separation between matter and DM and DE.

The non-equilibrium dynamics described here emerged by filling of the infinite number vacuum states that became emptied at energies above $E_{c}$. When this part of the filled vacuum condensed it generated a quartic potential in which the coupling $g$ and scale $a$ became microscopic asymmetric functions of the quark and antiquark densities. By inclusion of quantum effects, the coupling $g$ then gave a matter to DM ratio and DE density at correct orders of magnitude. The model also gave a realistic value of the cosmological "constant", $\Lambda=0.67$, corresponding to the $\mathrm{DE}$ density that determines the rate of inflation in the Universe [16]-[22]. At lower energies, the obtained microscopic quartic potential agreed with the quartic potential in EW theory. The model thus provides a form of direct link between the Universe immediately after the start of Big Bang at infinite high energy and high energy particle physics described by the SM.

The masses of neutrinos are usually described by a mixing of flavours in different seesaw models [23]-[30]. However, also neutrino quantum fields require a sufficiently filled vacuum. It would be interesting to see if the suggested model, which should also be able to explain the generation of surplus neutrinos, could help to discriminate between the various seesaw models.

\section{Conflicts of Interest}

The author declares no conflicts of interest regarding the publication of this paper.

\section{References}

[1] Kuzmin, V.A., Rubakov, V.A. and Shaposhnikov, M.E. (1985) Physics Letters B, 155, 36-42. https://doi.org/10.1016/0370-2693(85)91028-7

[2] Cohen, A.G., Kaplan, D.B. and Nelson, A.E. (1993) Annual Review of Nuclear and Particle Science, 43, 27-70. https://doi.org/10.1146/annurev.ns.43.120193.000331

[3] Carena, M., Quiros, M., Riotto, A., Vilja, I. and Wagner, C.E.M. (1997) Nuclear Physics B, 503, 387-404. https://doi.org/10.1016/S0550-3213(97)00412-4

[4] Kubo, J., Lim, K.S. and Lindner, M. (2014) Physical Review Letters, 113, Article ID: 091604. https://doi.org/10.1103/PhysRevLett.113.091604

[5] Ballesteros, G., Redondo, J., Ringwald, A. and Tamarit, C. (2017) Physical Review Letters, 118, Article ID: 071802. https://doi.org/10.1103/PhysRevLett.118.071802

[6] Ipek, S. and Tait, T.M.P. (2019) Physical Review Letters, 122, Article ID: 11200. https://doi.org/10.1103/PhysRevLett.122.112001

[7] Carena, M., Quiros, M. and Zhang, Y. (2019) Physical Review Letters, 122, Article ID: 201802. https://doi.org/10.1103/PhysRevLett.122.201802

[8] Ade, P.A.R., et al. 2021) Physical Review Letters, 127, Article ID: 151301. https://doi.org/10.1103/PhysRevLett.127.151301

[9] Dirac, P.A.M. (1930) Proceedings of the Royal Society of London, 126, 360. https://doi.org/10.1098/rspa.1930.0013

[10] Matsson, L. (2016) World Journal of Mechanics, 6, 441-445. https://doi.org/10.4236/wjm.2016.611031 
[11] Buttazo, D., Degrassi, G., Giardino, P.P., Giudice, G.F., Sala, F., Salvio, A. and Strumia, A. (2013) JHEP, 12, 89. https://doi.org/10.1007/JHEP12(2013)089

[12] Green, D. and Porto, R.A. (2020) Physical Review Letters, 124, Article ID: 251302. https://doi.org/10.1103/PhysRevLett.124.251302

[13] Hu, W., Spergel, D.N. and White, M.J. (1997) Physical Review D, 55, 3288. https://doi.org/10.1103/PhysRevD.55.3288

[14] Maldacena, J. (2016) Fortschritte der Physik, 64, 10-23. https://doi.org/10.1002/prop.201500097

[15] Matsson, L. and Meuldermans, R. (1977) Physics Letters B, 70, 309-312. https://doi.org/10.1016/0370-2693(77)90665-7

[16] Guth, A. (1981) Physical Review D, 23, 347-356. https://doi.org/10.1103/PhysRevD.23.347

[17] Linde, A.D. (1982) Physics Letters B, 108, 389-393. https://doi.org/10.1016/0370-2693(82)91219-9

[18] Albrect, A. and Steinhardt, P.J. (1982) Physical Review Letters, 48, 1220-1223. https://doi.org/10.1103/PhysRevLett.48.1220

[19] Bezrukov, F. and Shaposhnikov, M. (2008) Physics Letters B, 659, 703-706. https://doi.org/10.1016/j.physletb.2007.11.072

[20] Bezrukov, F., Kalmykov, M.Y., Kniehl, B.A. and Shaposhnikov, M. (2012) JHEP, 10, 140. https://doi.org/10.1007/JHEP10(2012)140

[21] Kanemura, S., Matsui, T. and Nabeshima, T. (2013) Physics Letters B, 723, 126-131. https://doi.org/10.1016/j.physletb.2013.05.002

[22] Okada, N. and Shafi, Q. (2015) Physics Letters B, 747, 223-228. https://doi.org/10.1016/j.physletb.2015.06.001

[23] Fukuda, Y., et al. (1998) Physical Review Letters, 81, 1562-1567.

[24] Minkowski, P. (1977) Physics Letters B, 67, 421-428. https://doi.org/10.1016/0370-2693(77)90435-X

[25] Gell-Mann, M., Ramond, P. and Slansky, R. (1979) Conf. Proc. C, 315, Article ID: 790927.

[26] Yanagida, T. (1980) Progress of Theoretical Physics, 64, 1103-1105. https://doi.org/10.1143/PTP.64.1103

[27] Fukugita, M. and Yanagida, T. (1986) Physics Letters B, 174, 45-47. https://doi.org/10.1016/0370-2693(86)91126-3

[28] Ipek, S., Plascencia, A.D. and Turner, J. (2018) JHEP, 12, 111. https://doi.org/10.1007/JHEP12(2018)111

[29] Dror, J.A., Hiramatsu, T., Kohri, K., Murayama, H. and White, G. (2020) Physical Review Letters, 124, Article ID: 041804. https://doi.org/10.1103/PhysRevLett.124.041804

[30] Greenberg, O.W. (1964) Physical Review Letters, 13, 598-602. https://doi.org/10.1103/PhysRevLett.13.598 